\title{
Cheap and cheerful circuits
}

\author{
Silicon remains the computer chip industry's material of choice. But for \\ simpler circuitry, it could soon have some surprising rivals. David Voss talks \\ to the scientists who are trying to make electronics go organic.
}

$t$ is easy to get the impression that research in electronics is all about pushing the limits of information processing. The fanfare that greets the launch of each new, ultrapowerful computer chip certainly makes it seem that way. But another trend is quietly gathering force: a push to exploit the modest end of cost and performance. For the past few years, materials scientists and device engineers have been collaborating to develop a new breed of cheap and cheerful electronic components. Their working medium: not silicon, nor metals — but plastics and smaller organic molecules.

The goal of organic electronics is to make devices that are cheap, flexible and just smart enough to perform the task in hand. Electronic price tags, flexible computer screens and disposable cell phones are among the potential applications. Their components will be created not in billion-dollar fabrication facilities, like today's silicon chips, but using printing technologies little more sophisticated than those found in a desk-top inkjet printer.

"People talk about microelectronics," says materials scientist Francis Garnier of the CNRS Laboratory of Molecular Materials in Thais, part of France's national research agency. "But you can also have macroelectronics - low-end and inexpensive." And in the past year, several groups have announced striking results that have boosted the field's prospects. "It's been a rich haul," says Ananth Dodabalapur, whose team at Lucent Tech-

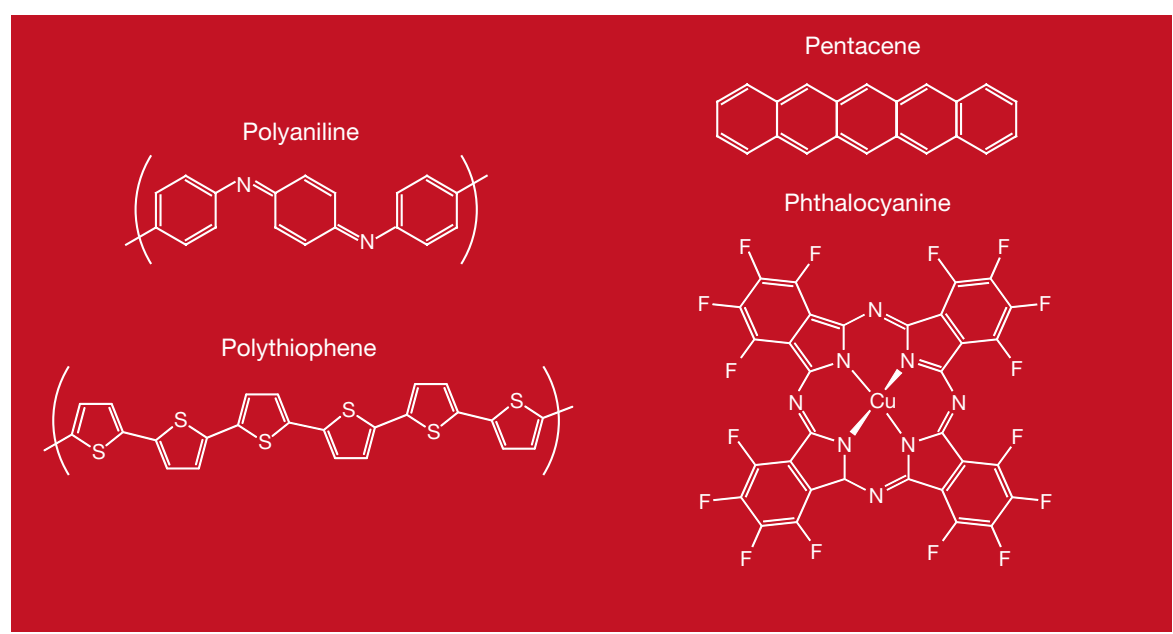

Conjugal bliss: polymers (left) and smaller organic molecules (right) that can act as conductors or semiconductors have a 'conjugated' structure of alternating single and double bonds.
An early hint of the possibilities offered by plastic electronics came in 1994, when Garnier's group reported making the first all-polymer transistors ${ }^{1}$. Laying down the molecules using simple printing technology, rather than sophisticated techniques such as high-vacuum deposition or photolithography, Garnier and his colleagues fashioned rudimentary transistors out of the organic semiconductor polythiophene. Organics and polymers had been used in transistors previously, but this was the first time transistors - including their electrodes - had been made completely from plastic. As a result, the transistors kept on working even when the substrate was bent through angles of $90^{\circ}$.

\section{Towards integration}

But transistors are not much use by themselves. The next step is integration - linking many transistors together to process information. Dago de Leeuw and his colleagues at Philips Research Laboratories in Eindhoven, the Netherlands, took the first big step forward in 1998, producing a plastic integrated circuit in which batches of transistors were hooked together into a functional unit ${ }^{2}$. After making individual transistors, and then simple logic gates, the researchers eventually fashioned a 15-bit code generator containing 326 all-polymer transistors. This is a device that creates a unique digital code for use as an identifying number, rather like a barcode. And it has already found a commercial niche: Philips has incorporated the circuit into identification tags that are used as anti-theft stickers for retail goods.

De Leeuw and his colleagues achieved their results using a kind of photochemical patterning. First, they coated a substrate with a mixture of polyaniline and a photochemically reactive chemical, spinning the substrate rapidly so that the coating materials ended up as a film less than one micrometre thick. The photoreactive compound had the handy property of turning the normally conductive polyaniline into an insulator when exposed to ultraviolet radiation. So when the substrate was exposed to ultraviolet light through a mask, the irradiated parts of the film underwent a chemical transformation that boosted electrical resistance by 11 orders of magnitude. With the basic geometry of the circuit laid down in 


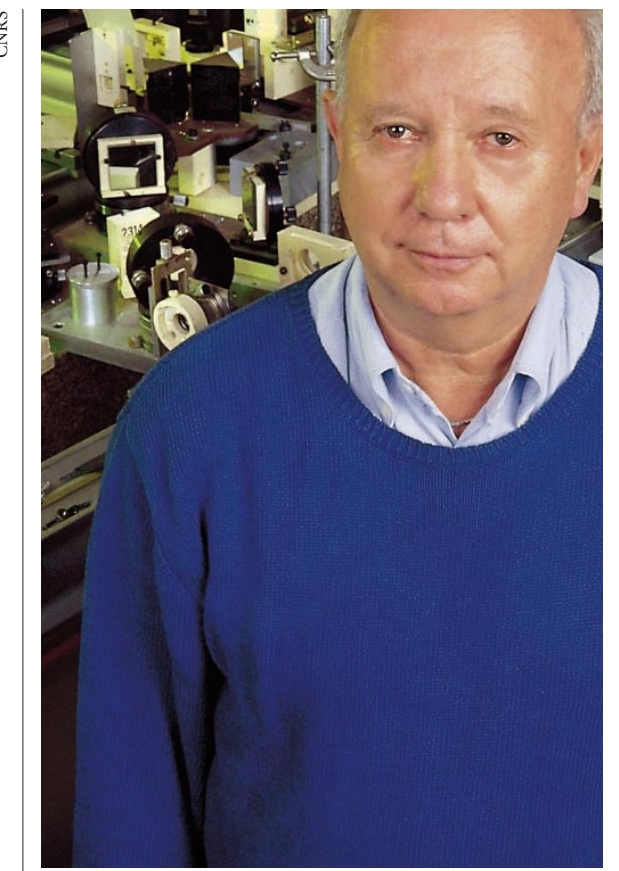

Garnier: trying to make cheap "macroelectronics".

this way, the researchers deposited a film of their organic semiconductor, polythienylenevinylene, followed by an insulating layer of polyvinylphenol. Finally, the researchers punched tiny holes through the substrate, into which polyaniline seeped, connecting the output of one transistor within the circuit to the input of the next.

In February this year, Dodabalapur and his colleagues at Lucent raised the bar ${ }^{3}$. Their circuits are not made entirely of polymers the component transistors are connected using nickel and gold. But they use much less power than the Philips device, thanks to a technique called complementary logic, borrowed from conventional silicon microelectronics. Semiconductors come in two flavours, n- and p-type, depending on whether they have a surplus or a deficit of electrons. Transistors can be made out of either, but device engineers discovered long ago that circuits made only of $n$ - or p-type transistors draw lots of current. Logic elements made from a combination of the two, on the other hand, draw very little except when they are switched on or off.

\section{From science to gadgets}

Taking a complementary-logic approach, the Lucent team created circuits containing 864 organic transistors. They used two different organic semiconductors - a phthalocyanine for the n-type elements and a thiophene for the p-type - on a flexible plastic substrate. The fanciest gadget they have made is a shift register, a ubiquitous digital circuit that shifts bits from a set of inputs to a row of outputs in an orderly fashion - microprocessors use them to do arithmetic and video cameras use them to scan pixels for incoming light. In the jargon

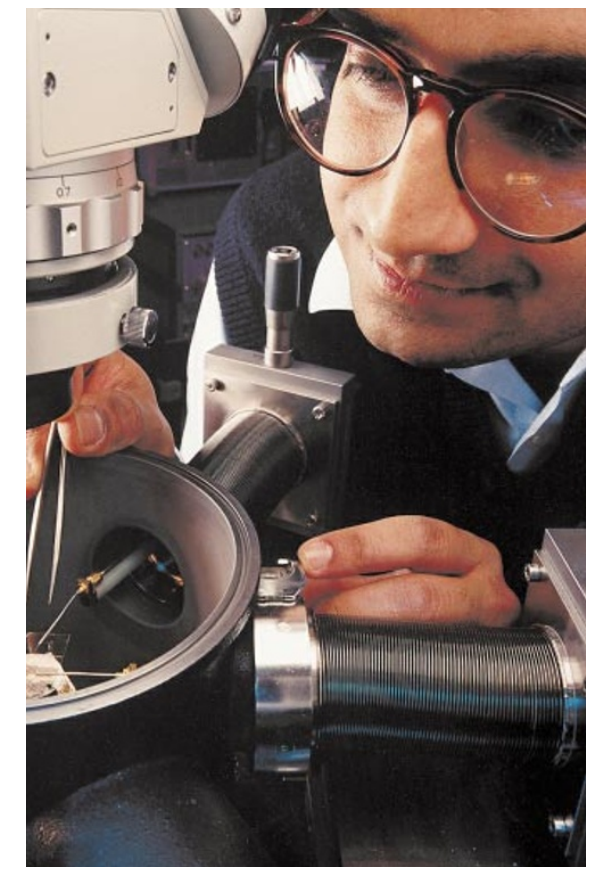

Dodabalapur: mixing the organic and inorganic.

of digital engineering, this circuit is approaching 'large-scale integration', meaning that a single chip contains thousands of components. For comparison, Intel's Pentium processors are now into the realm of ultralarge-scale integration, with almost ten million transistors etched onto a single chip. But the proponents of organic electronics note that many applications do not need this enormous processing power.

Not to be outdone, Philips has applied polymer electronics to display technology. The screen on a laptop personal computer looks crisp because it uses active-matrix technology, in which each pixel is controlled by its own transistor switch. In today's liquid-crystal screens, these are made from amorphous silicon. But the cost and limits to fabricating this silicon mean that it is not economically viable to build screens

larger than about $38 \mathrm{~cm}$ across.

Philips has now created a crude active-matrix display using polymer transistors. Although this is a demonstration device measuring just 64 by 64 pixels, polymer electronics offers the possibility of building large yet affordable liquidcrystal displays.

De Leeuw's group at Philips also returned this month with an increase in the performance of their all-polymer integrated circuits ${ }^{4}$. The key was using materials with a higher carrier mobility. This is an important attribute for any semiconducting material. Crudely put, mobility is a measure of how fast an electron or hole - the absence of an electron - can travel through a material. In practical terms it limits how quickly a transistor can switch on and off. Since even low-end electronics need to switch reasonably quickly, improving mobility is important. In their latest design, the Philips researchers use high-mobility organic semiconductors such as pentacene and hexylthiophene. So whereas the group's earlier circuits could only operate at switching rates up to about 200 hertz, the new versions operate at several kilohertz.

\section{Upward mobility}

The message here is that materials science is the key to the future development of organic electronics - find a better organic semiconductor and improved circuits will follow. Right now, the polymers that are easiest to build into electronic circuits have frustratingly low carrier mobility - about a million times lower than silicon. The problem is that there tends to be a trade-off between an organic molecule's mobility and the ease with which it can be processed. Because the molecular bonding in organic molecules is relatively weak, they tend to be mechanically flexible and soluble, which makes devices easy to fabricate by liquid deposition or the Philips group's spincoating technique.

But the weak bonding also means that electrons hop from molecule to molecule relatively slowly. And attempts to boost mobility by strengthening bonding also increase the stiffness of the material and reduce its solubility. Organic molecules such as pentacene may have much better mobility, but devices constructed from them are more difficult to manufacture and more expensive. Other high-tech organic

Flexible friend: some organic circuits such as this polyimide foil - will work even when bent. 


\section{news feature}

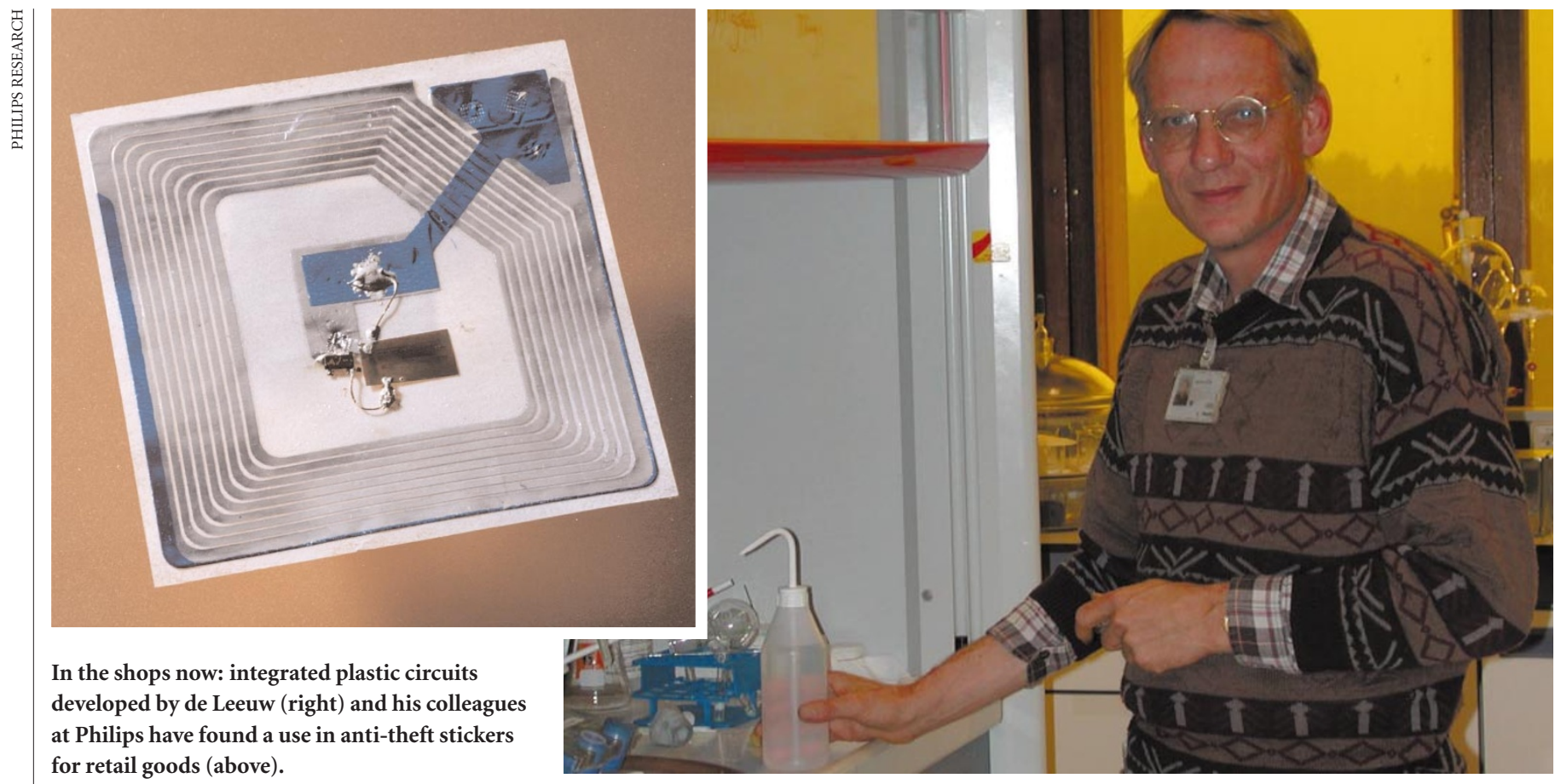

- semiconductors degrade when exposed to the air.

Even so, those with experience of researching the properties of organic materials are confident that the problems can be solved. Cambridge's Friend believes that the key to improving mobility is to lay down organic molecules in ordered arrays, rather than spaghetti-like tangles. "You can arrange to get highly ordered structures if you have the right recognition between the substrate and polymer being laid down from solution," he says.

Studies of polythiophenes by Friend's group have already shown that highly ordered structures have improved mobility. The researchers deposited these polymers onto a substrate made of a mixture of silicon and silicon oxide so that the molecules organized themselves into ordered crystalline sheets either parallel or perpendicular to the surface. They discovered that the orientation affected carrier mobility, with this being two orders of magnitude higher in the parallel crystals than in the perpendicular ${ }^{5}$.

More recently, the Cambridge team has worked out how to lay down films of polyfluorene that assume a liquid-crystal state and become highly ordered ${ }^{6}$. This enhances the material's carrier mobility by a factor of about ten. And because much is already known about how to attain order within liquid crystals, this approach could provide a means to improve the carrier mobility of a range of organic semiconductors. "These are the sort of approaches that can be pushed much further," says Friend.

Another promising approach is to make hybrid materials - composites of organic and inorganic molecules that combine the benefits of each and, it is hoped, leave the drawbacks behind. Christos Dimitrakopoulos and his group at IBM's Thomas J. Watson Research Center in Yorktown Heights, New York, have been studying molecular-scale composites that combine the properties of inorganic crystalline materials with those of organic molecules ${ }^{7}$. The idea is to retain the mobility of the inorganic components and make them easier to process by gluing them together, so to speak, with the organics.

\section{The perovskite cookbook}

The IBM team has been working with perovskites, in which inorganic molecules are linked together to form an extended layered framework. One class of perovskites has already attracted lots of attention as hightemperature superconductors, and it is well known that the materials' electronic properties can be tuned. Dimitrakopoulos and colleagues stirred together the inorganic compound tin iodide, which has a respectable carrier mobility, with the organic compound phenethyl ammonium. Then they spun-coated the mixture onto a substrate. As it dried, the composite material self-assembled to form multiple sheets of octahedral tin iodide molecules with organic layers sandwiched in between.

The resulting perovskite had a mobility of up to 0.6 square centimetres per volt second, six times higher than any other spin-coated material. Although not in the same class as silicon in terms of mobility, the layered perovskite beats silicon hands down in processability. Dimitrakopoulos expects that with some work, the material's carrier mobility might be pushed as high as 50 square centimetres per volt second, approaching that of pure tin iodide. Moreover, the perovskite could be fashioned into both $\mathrm{p}$ - and n-type

^ ๔ 2000 Macmillan Magazines Ltd semiconductors - and so offers the hope of manufacturing low-cost complementary logic circuits.

Other researchers are improving the potential performance of organic electronic devices by concocting strongly insulating materials that can be laid down in very small spaces, which will prevent current leaking out of organic transistors and their electrodes. For example, Dominique Vuillaume and his group at the Northern Institute of Electronics and Microelectronics in Villeneuve d'Ascq, France, have been making extremely thin insulating layers for use in organic transistors. Lately, they have managed to self-assemble monolayers of alkyltrichlorosilanes just two nanometres thick that function as the gate insulator in a fieldeffect transistor ${ }^{8}$.

With materials scientists continuing to coax better performance out of organic electronic devices, the prospects for the field should continue to brighten. As yet, it is too early to predict the eventual scope of the plastic electronic revolution. But with Philips' anti-theft stickers already in use, and its active-matrix display at the prototype stage, the first of silicon's barricades have already been stormed.

David Voss is a science writer in Silver Spring, Maryland.

1. Garnier, F., Hajlaoui, R., Yassar, A. \& Srivastava, P. Science 265, 1684-1686 (1994)

2. Drury, C., Mutsaers, C., Hart, C., Matters, M. \& de Leeuw, D Appl. Phys. Lett. 73, 108-110 (1998).

3. Crone, B. et al. Nature 403, 521-523 (2000).

4. Gelinck, G., Geuns, T. \& de Leeuw, D. Appl. Phys. Lett. 77, 1487-1489 (2000).

5. Sirringhaus, H. et al. Nature 401, 685-688 (1999)

6. Sirringhaus, H. et al. Appl. Phys. Lett. 77, 406-408 (2000).

Kagan, C. R., Mitzi, D. B. \& Dimitrakopoulos, C. D. Science 286, 945-947 (1999).

8. Collet, J., Tharaud, O., Chapoton, A. \& Vuillaume, D. Appl. Phys. Lett. 76, 1941-1943 (2000)

NATURE |VOL 407| 28 SEPTEMBER 2000 | www.nature.com 